\title{
ESTUDIO DEL COMPORTAMIENTO DE LA ESCAYOLA REFORZADA CON FIBRAS DE SISAL, PARA COMPONENTES EN VIVIENDAS DE BAJO COSTE
}

\author{
(STUDY OF THE BEHAVIOUR OF SISAL FIBER REINFORCED HEMIHYDRATED \\ GYPSUM AS COMPONENTS IN LOW COST HOUSING)
}

Ignacio de Oteiza San José, Dr. Arquitecto

Universidad Politécnica de Madrid,

Escuela Técnica Superior de Arquitectura.

ESPAÑA

\section{RESUMEN}

El presente artículo es una breve descripción de los trabajos realizados para la elaboración de la tesis doctoral $y$ de las conclusiones de la misma.

Partiendo de las posibilidades de utilización en los países en vias de desarrollo del material de escayola, por su abundancia $y$ bajo coste, se llevan a cabo una serie de ensayos mecánicos, con el fin de conocer las propiedades fisicas y mecánicas de la escayola reforzada con fibras de sisal, para una futura

aplicación en componentes para viviendas de bajo coste.
Director de Tesis:

Luis de Villanueva y Domínguez, Dr. Arquitecto.

Fecha de recepción: 2-XI-93

\section{SUMMARY}

The present article is a brief description of the work done for the elaboration of the doctoral thesis and its conclusions.

Starting from the possibilities of using plaster in developing countries, due to its abundance and low cost, a series of mechanical tests have been carried out in order to learn about the physical and mechanical

properties of plaster reinforced with sisal fibres for its future use in components for low cost housing.

\section{INTRODUCCIÓN}

El objetivo de esta tesis es el estudio teóricoexperimental de la escayola reforzada con fibras cortas de sisal, con el fin de facilitar la utilización de este material en la realización de componentes constructivos para viviendas de bajo coste.

La necesidad de cantidad y calidad de viviendas en los países en vías de desarrollo, hace necesaria la búsqueda, mejora y utilización de materiales de bajo coste que permitan una fácil aplicación en las viviendas.

El yeso ha sido utilizado desde hace mucho tiempo, siendo uno de los primeros conglomerantes que utilizó el hombre. Tiene gran variedad de aplicaciones en la construcción, desde su uso para revestimientos, hasta su utilización en la elaboración de elementos prefabricados. Aunque el yeso o la escayola han sido utilizados conjuntamente con fibras vegetales, siendo ésta una práctica tradicional, el comportamiento mecánico de este compuesto, así como los mecanismos que actúan entre las dos fases, requieren de un análisis más profundo. Por otro lado, en muchos países en vías de desarrollo, la utilización del yeso y la escayola es muy limitada, a pesar de contar, en la mayoría de estos países, con reservas de mineral de yeso, pudiendo ser explotado y transfơrmado en material y componente constructivo, con menores inversiones, que otros materiales de mayor demanda para la construcción con viviendas. 
FLEXOTRACCIONN (UNE 102 031-82).

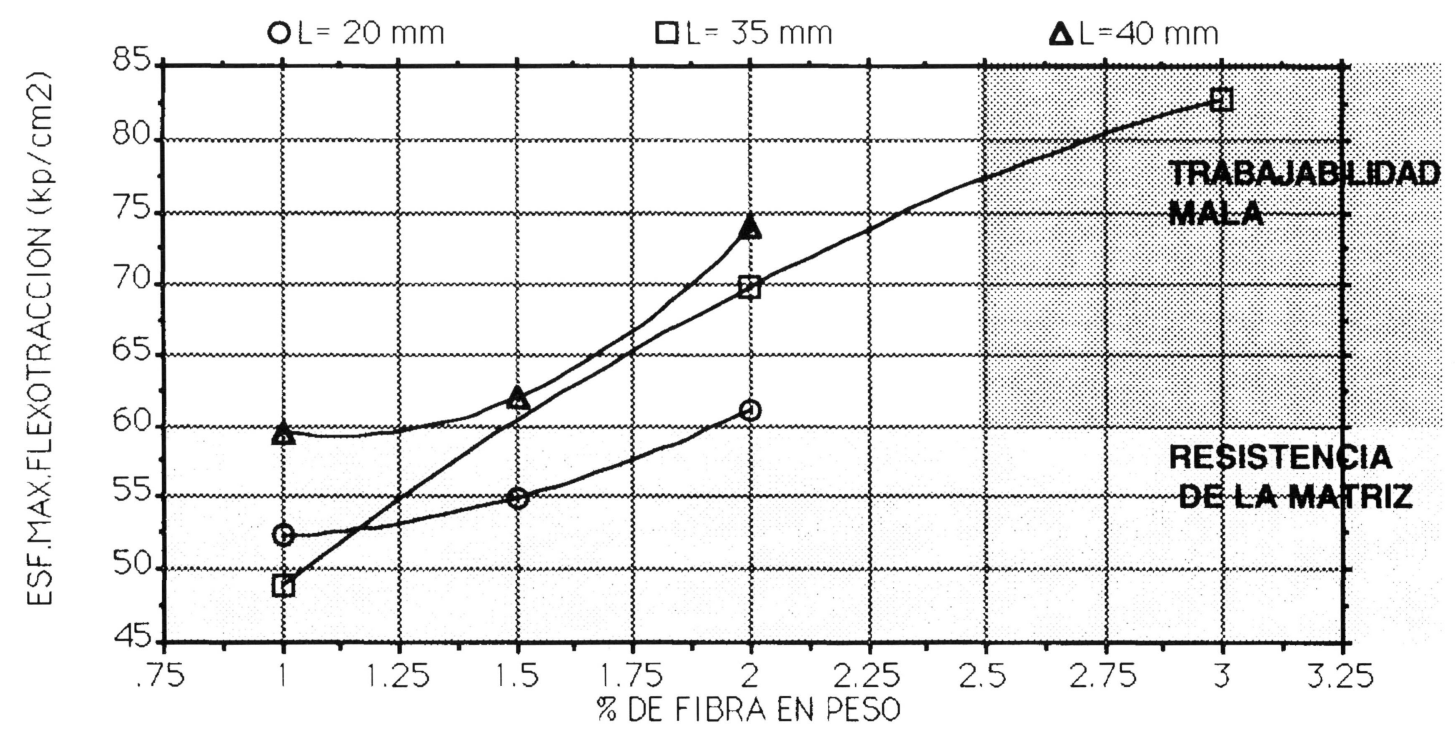

Fig. 1.- Resistencia a flexotracción del compuesto. Con diferentes longitudes $(20,35$ y $40 \mathrm{~mm})$ y cantidades de fibras.

Se desarrolla el estudio experimental en dos etapas:

- Una primera parte dedicada al estudio de los materiales que conforman el compuesto, la escayola como matriz y las fibras de sisal como refuerzo, con el fin de determinar el comportamiento mecánico de cada uno de estos materiales para seleccionar su composición al utilizarlos conjuntamente.

-La siguiente parte, está dirigida al estudio experimental sobre el comportamiento físicomecánico de la escayola reforzada con adiciones de fibras cortas de sisal. La finalidad al realizar este material es buscar mejoras en algunas características mecánicas de la escayola, para poder hacer uso de este material en la prefabricación de componentes de viviendas.

El proceso experimental se lleva a cabo analizando el comportamiento del material compuesto, mediante el estudio de diferentes variables, tales como: agua de amasado, longitud y cantidad de fibras. Mediante ensayos se determinan: las resistencias a compresión, flexotracción e impacto, el módulo de elasticidad, la tenacidad y la adherencia entre las fibras y la matriz, lo que permite conocer el comportamiento mecánico del material e indicar las composiciones más recomendables para la elaboración de elementos constructivos con este material.

(c) Consejo Superior de Investigaciones Científicas
Por otro lado, conociendo el buen comportamiento del yeso y de la escayola a altas temperaturas, se lleva a cabo un estudio experimental, en pequeños elementos de escayola reforzada con fibras de sisal, determinando las mejoras que aportan las fibras cuando el material está sometido a fuego.

\section{BASES DE PARTIDA}

De los tres primeros capítulos destacan las siguientes bases de partida:

- Ante la grave crisis del sector habitacional, en los países en vías de desarrollo, es necesario promover la utilización de materiales abundantes de bajo coste, que puedan ser utilizados en la elaboración de diferentes componentes constructivos para viviendas de bajo coste.

La incidencia de los costes de los materiales de construcción, en las viviendas de los sectores poblacionales de bajos recursos, llega a representar del orden del $\mathbf{6 4 \%}$ del coste total de la construcción.

- El mineral de yeso es un material abundante en el mundo, de fácil explotación y cłya trasformación en material para la construcción no implica grandes inversiones en instalaciones industriales, y demanda menos cantidad de energía que la 


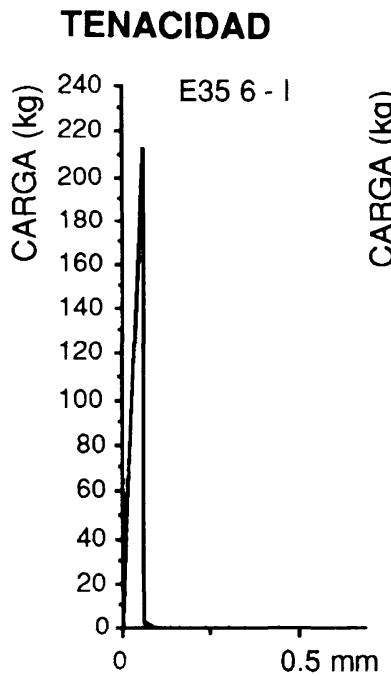

(a)

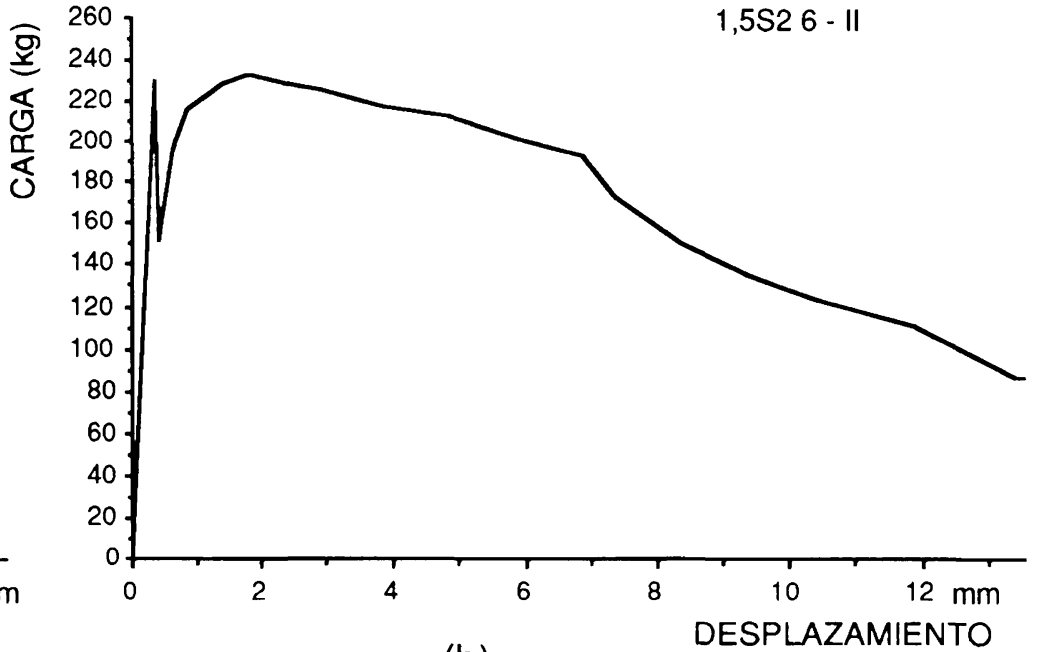

(b)

Fig. 2.- Curvas de carga-desplazamiento: (a) Escayola sin fibras. (b) Escayola reforzada con fibras cortas de sisal $(1,5 \%-L=20 \mathrm{~mm})$.

mayoría de los materiales tradicionalmente utilizados en la construcción de viviendas.

El yeso y la escayola son materiales que pueden sustituir al cemento en partidas de obra importantes de la vivienda, siendo su coste menor que el del cemento (alrededor del $50 \%$ según las zonas).

- Las prestaciones que ofrece el yeso o la escayola, referentes a la habitabilidad, tales como confort higrotérmico, resistencia al fuego, aislamiento térmico y acústico, entre otros, constituyen un factor importante de mejora en la calidad de las viviendas.

- Por su fácil moldeabilidad y su reducido peso, permite la prefabricación de componentes constructivos que pueden ser aplicados en desarrollos de viviendas, tanto en procesos industrializados, como en autoconstrucción.

- Uno de los aspectos que dificulta la utilización del yeso en la construcción de viviendas, es su comportamiento de rotura de tipo frágil, lo que impide su aplicación en componentes constructivos expuestos a golpes o impactos durante la construcción, manipulación y vida útil.

\section{- El yeso mediante la adición de fibras mejora} parte de sus propiedades físicas y mecánicas y de manera significativa lo relativo a su fragilidad.
- La adición de fibras artificiales, como la fibra de vidrio al yeso o la escayola, representa un incremento de los costes de los materiales y componentes, llegando a ser del orden del $25 \%$, en países importadores de fibras artificiales.

- Las fibras vegetales han sido tradicionalmente utilizadas en la construcción de viviendas en muchos países. La utilización de fibras naturales como alternativa a algunas fibras artificiales, en países en vías de desarrollo, representa un ahorro de energía; por su abundancia y variedad son económicas.

- El sisal (fibra de la agave sisalana, conocida en España como pita), es una fibra que se extrae de una planta muy extendida por todo el mundo, abundando especialmente en América Latina, Africa y los países mediterráneos.

La mezcla de escayola con fibras vegetales, y específicamente con el sisal, no presenta reacciones de tipo químico, que puedan afectar a alguno de los dos materiales.

\section{METODOLOGÍA Y RESULTADOS}

Para estudiar el comportamiento del material compuesto de escayola reforzada con fibras de sisal, se llevan a cabo diferentes ensayos de cada uno de los materiales que conforman el compuesto 


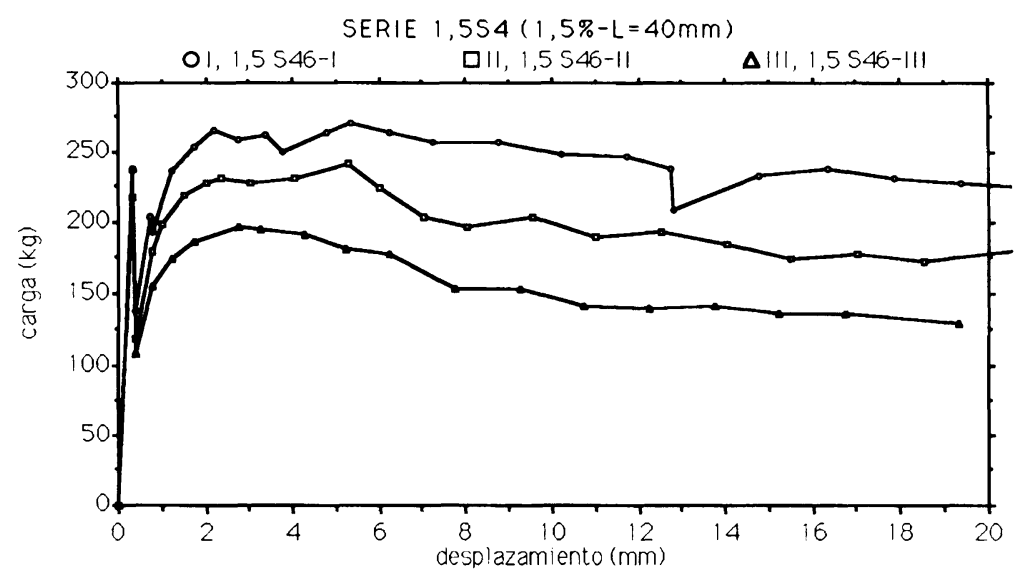

Fig. 3.- Diagrama carga-desplazamiento de la serie 1,554.

\section{TENACIDAD}

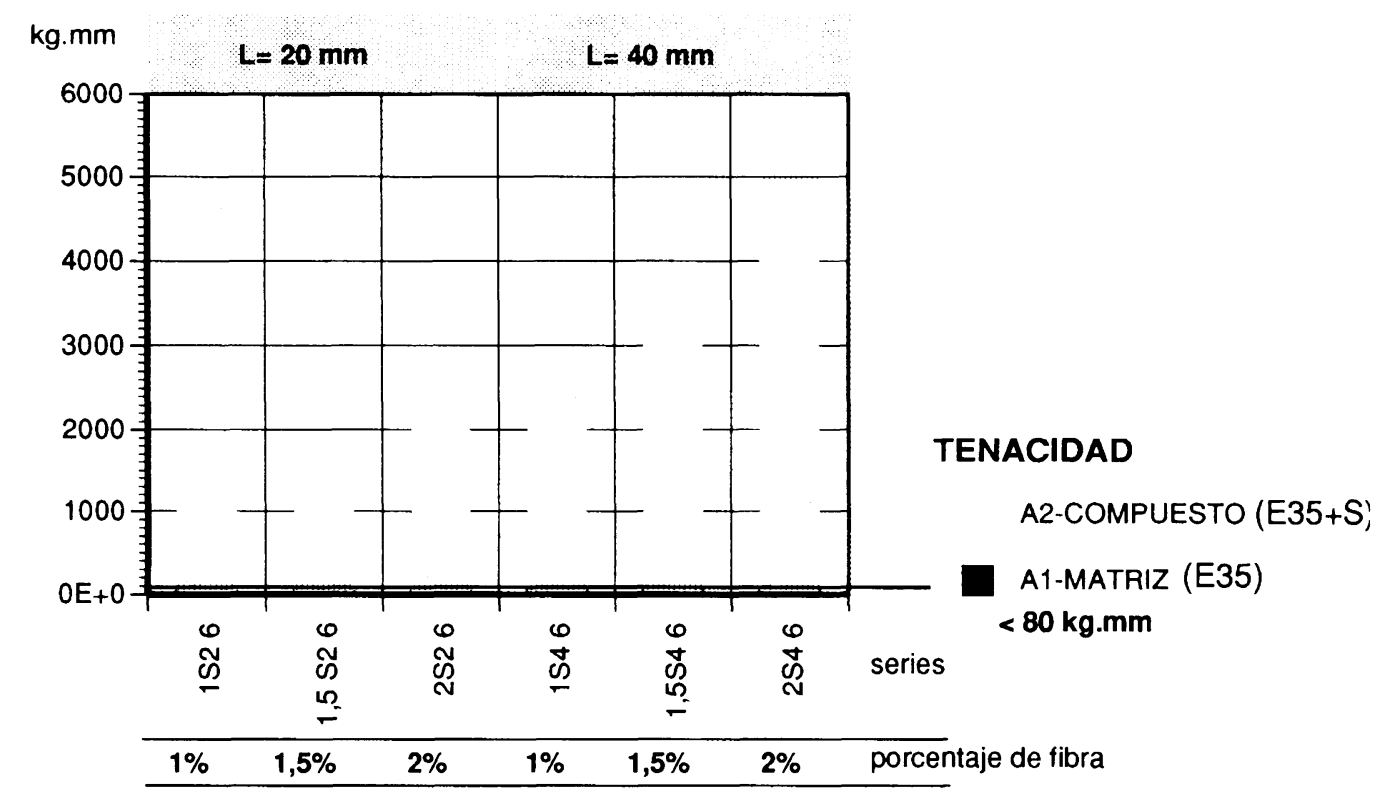

Fig. 4.- Relación entre la tenacidad de la matriz (escayola) y del compuesto (escayola reforzada con fibras). Variando la longitud y la cantidad de fibras.

(escayola y fibras de sisal), para determinar sus características y posteriormente proceder a la realización de los ensayos del material compuesto. Alguno de estos ensayos (compresión, flexotracción) están establecidos en la norma UNE 102.030-82; otros, como adherencia, tiempos de fraguado, comportamiento ante el fuego e impacto, se diseñaron para la realización de este trabajo.

A continuación se presentan los resultados de los ensayos más significativos.

\section{CONCLUSIONES}

\section{A.- Del estudio de la escayola reforzada con sisal}

Las mejoras más importantes del compuesto, en relación con la escayola sin reforzar, están representadas por el incremento de la tenacidad en los ensayos de flexotracción y la capacidad de absorción de energía en los ensayos de impacto El comportamiento del compuesto, en relación con la rotura frágil de la escayola sin reforzar, 


\section{ADHERENCIA}

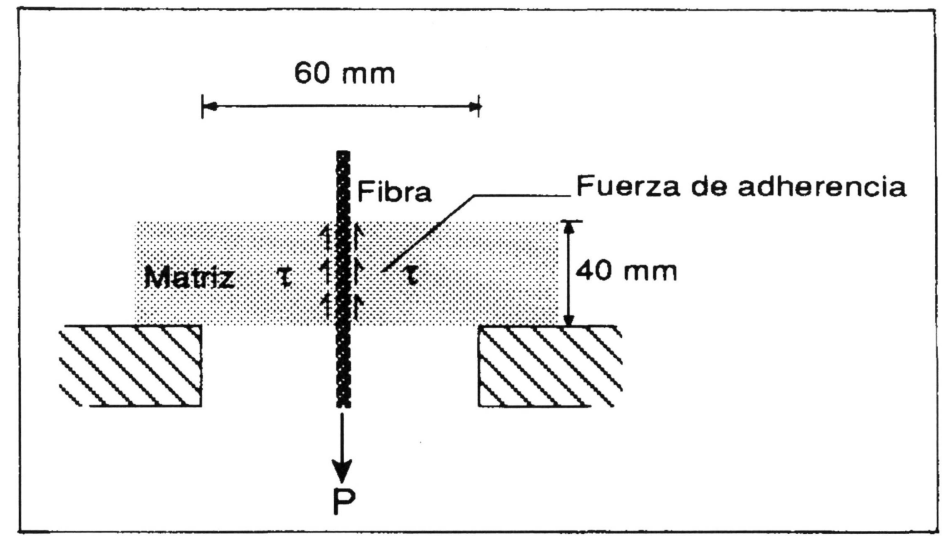

Fig. 5.- Ensayo de adherencia tipo A

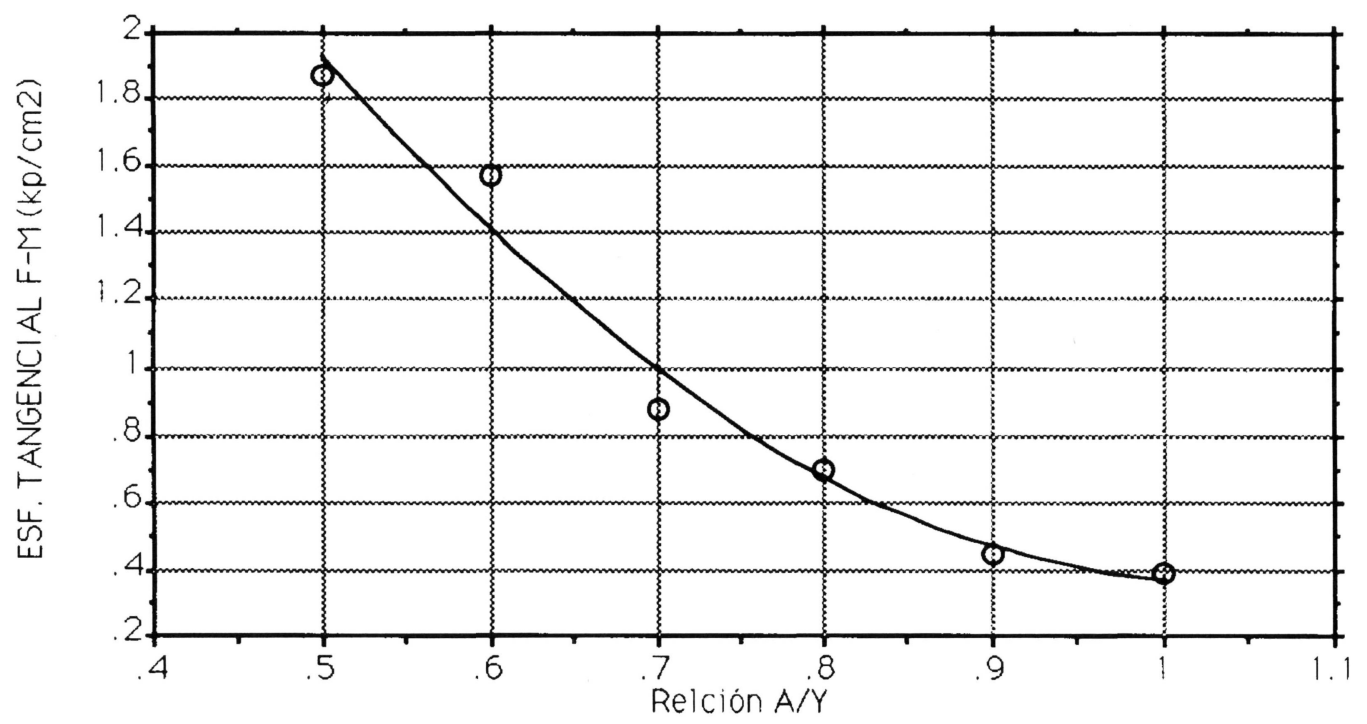

Fig. 6.- Determinación del esfuerzo de adberencia según la relación A/Y en la matriz.

cambia sustancialmente con la adición de fibras cortas de sisal, aumentando la tenacidad del material en función del incremento de la cantidad y de la longitud de las fibras.

El compuesto de la escayola reforzada con fibras cortas y dispersas en la matriz, que alcanza una mayor tenacidad, es el que corresponde a contenidos de fibra del $2 \%$ en peso y longitudes

(c) Consejo Superior de Investigaciones Científicas Licencia Creative Commons 3.0 España (by-nc) de $40 \mathrm{~mm}$, representando incrementos de la tenacidad del orden de $\mathbf{6 0}$ veces más que la tenacidad de la matriz.

Ante acciones de impacto, la escayola sin reforzar se rompe de manera frágil; con la adición de fibras de sisal se incrementa la resistencia del material a impacto, llegando a ser 40 veces superior a la de la escayola sin 


\section{ENSAYO DE IMPACTO}

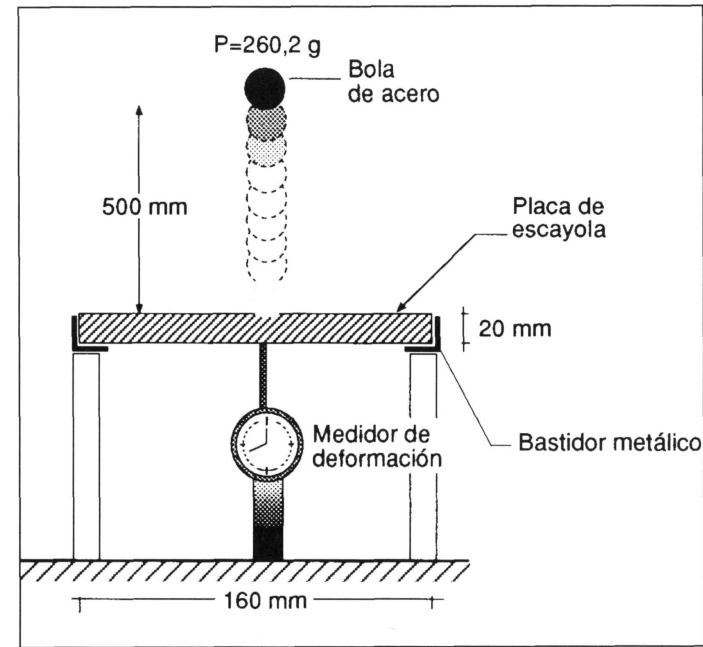

Fig. 7.- Disposición del ensayo de impacto.

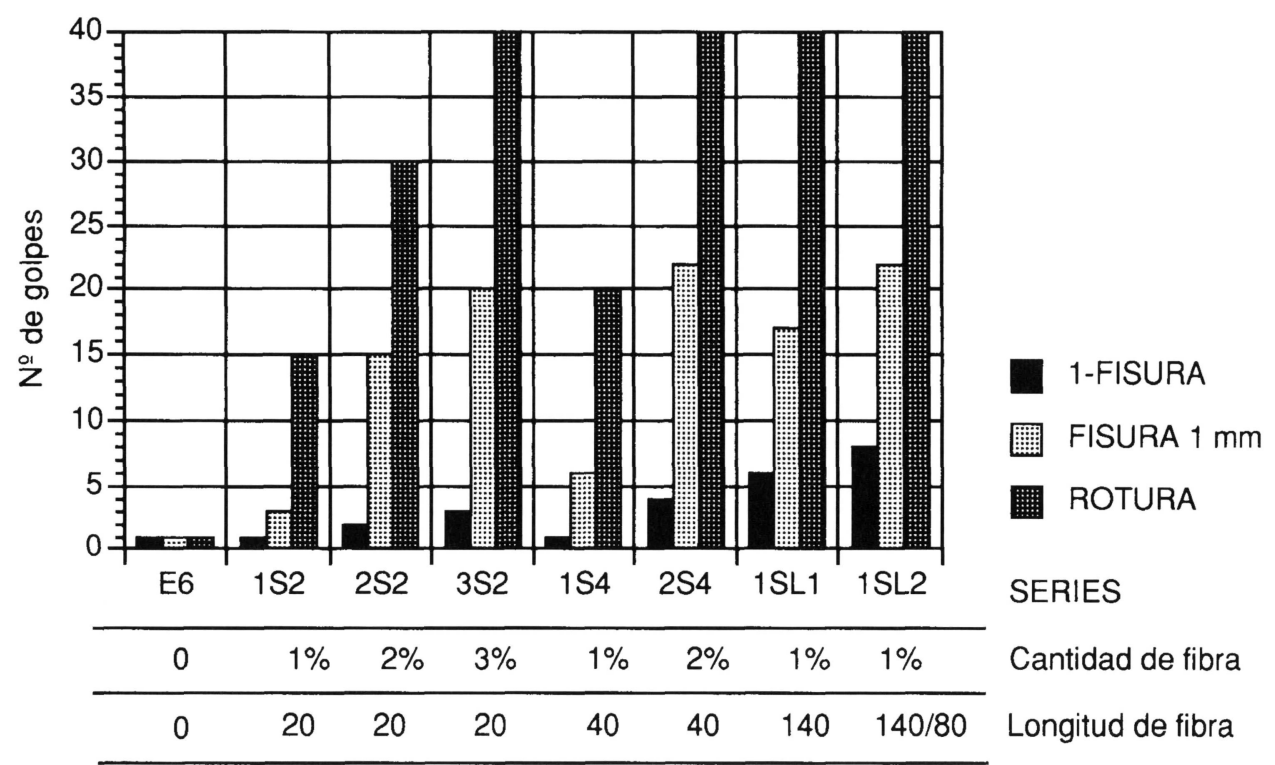

Fig. 8.- Resultados del ensayo de impacto de todas las series.

reforzar, debiéndose esto a la absorción de energía de las fibras incluidas en la matriz.

El aumento de la tenacidad en el yeso de flexotracción y de la resistencia a impacto del material reforzado, se debe fundamentalmente a la capacidad de adherencia entre la fibra y la matriz, permitiendo la transmisión de esfuerzos entre ambos materiales, mediante los micromecanismos de descohesión y arranque de las fibras, siendo el primer mecanismo mucho más efectivo para la mejora de la tenacidad.

\section{A-1.- Adherencia fibra-matriz}

Para escayolas con relación de agua-yeso de 0,6, la longitud crítica de la fibras de sisal está alrededor de los $\mathbf{4 0} \mathbf{~ m m}$; si se aumenta la cantidad de agua de amasado hasta $\mathrm{A} / \mathrm{Y}=0,8$, la longitud crítica de la fibra de sisal se incrementa en más del doble, Lc $=90 \mathrm{~mm}$. Esto confirma que cuanto más porosa es la escayola, su adherencia con las fibras-disminuye. Se ha determinado la siguiente ecuaєión:

$$
\tau_{\mathrm{f}-\mathrm{m}}=5,24(\mathrm{~A} / \mathrm{Y})^{2}-10,99(\mathrm{~A} / \mathrm{Y})+6,12
$$


que permite relacionar el esfuerzo tangencial de adherencia fibra-matriz, con la cantidad de agua de amasado en la matriz.

\section{A-2.-Resistencia a flexotracción}

La resistencia a flexotracción de la escayola reforzada con fibras cortas $(10-40 \mathrm{~mm})$ de sisal y dispersas en cantidades hasta de $2 \%$ en peso, no representa una mejora significativa en relación con la matriz sola, llegando la resistencia a ser un $\mathbf{2 0} \%$ superior a la de la matriz sola, cuando la longitud de la fibra es de $40 \mathrm{~mm}$ con $2 \%$ de cantidad de fibras.

Con la adición de fibras, de longitud menor que la crítica, se aprecia una reducción de la resistencia a flexotracción de la matriz, determinada por la primera fisura del material, que representa una disminución del 20\% de la resistencia de la escayola no reforzada. Este fenómeno se debe a un incremento de la porosidad en la matriz al mezclarla con las fibras de sisal.

El principal aporte del compuesto de escayola con sisal, en el ensayo de flexotracción, es el incremento de la ductilidad; el material presenta deformación plástica después de la primera fisura de la matriz. Las fibras confieren al material.

\section{B.- Aplicación del Compuesto}

De los estudios experimentales realizados, se deduce, que desde el punto de vista tecnológico es posible utilizar el compuesto de escayola reforzada con fibras de sisal, en la elaboración de componentes constructivos para viviendas, dado el buen comportamiento de este material ante acciones de impacto, su mayor tenacidad a flexotracción y resistencia ante el fuego.

\section{SUGERENCIAS PARA FUTUROS TRABAJOS DE INVESTIGACIÓN}

De la experiencia adquirida durante el desarrollo de este trabajo de investigación, y teniendo muy en cuenta el estado del arte del mismo, se considera que algunos temas o aspectos puntuales pueden estudiarse, con mayor profundidad, para obtener una mejor comprensión del material compuesto que facilite la aplicación práctica de estos estudios. Se señalan de forma esquemática las siguientes líneas de trabajo:
- La adición de las fibras de sisal a la escayola, debido a la trabajabilidad y con las técnicas de amasado utilizadas, presenta como límite máximo de adición de fibras un $2 \%$ en peso; dada la evidente mejora del compuesto, con el incremento de la cantidad de fibras, es interesante el estudio de otros sistemas que permitan añadir más fibras a la matriz, como por ejemplo la proyección de escayolas sobre entramados o tejidos de fibras o sistemas especiales de premezclado.

- Las probetas prismáticas de 40 x 40 x 160 mm presentan limitaciones, en cuanto al tamaño, cuando se requieren longitudes de fibras superiores a los $40 \mathrm{~mm}$, por lo que es conveniente investigar con elementos de mayor tamaño que permitan incrementar la longitud de las fibras para determinar su comportamiento mecánico. Por otro lado la elaboración de elementos constructivos de tamaño real, que puedan ser ensayados, permitirá evitar posibles desviaciones en los resultados que pueden deberse a los cambios de escala, resolviendo a la vez aspectos de tipo práctico como moldes, juntas entre los elementos, etc.

- Uno de los temas fundamentales de la escayola es el estudio de su porosidad, ya que esta característica determina su comportamiento mecánico. Cuando se utilizan fibras para reforzar la escayola, su adherencia está directamente relacionada con la porosidad de la matriz; se considera importante profundizar en este tema, buscando la mejora de la adherencia fibra matriz, mediante la reducción de porosidad. Es posible que a través de la compactación o de la reducción del agua de amasado se logre una mayor adherencia.

- Mediante los ensayos de fuego, se determinan en este trabajo algunas mejoras del material al estar reforzado, sin embargo estos ensayos han sido realizados en pequeñas probetas, de $20 \mathrm{~mm}$ de espesor, con el fin de conocer el comportamiento del compuesto a altas temperaturas; para reproducir algún componente de escayola reforzada con sisal, es necesario realizar los ensayos de fuego con el tamaño real del elemento, siendo muy probable que con espesores de placa mayores a los ensayados, las fibras de sisal en la escayola mejoren el comportamiento ante el fuego, por laque es conveniente continuar con este tema. 


\section{DETERMINACIÓN DE LOS TIEMPOS DE FRAGUADO (Técnica de Ultrasonido)}

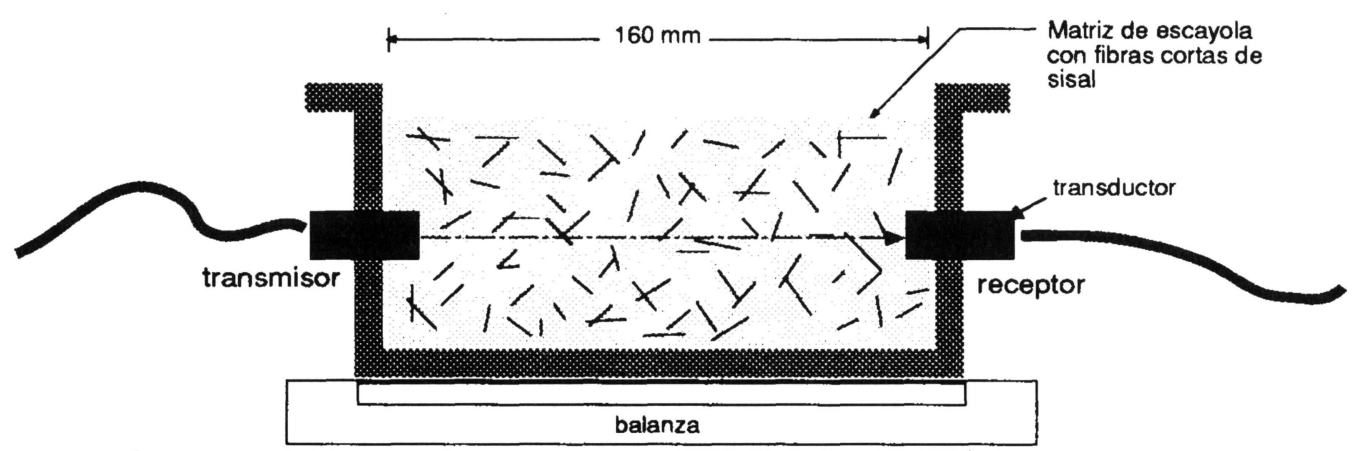

Fig. 9.- Molde y traductores del ensayo de ultrasonido.

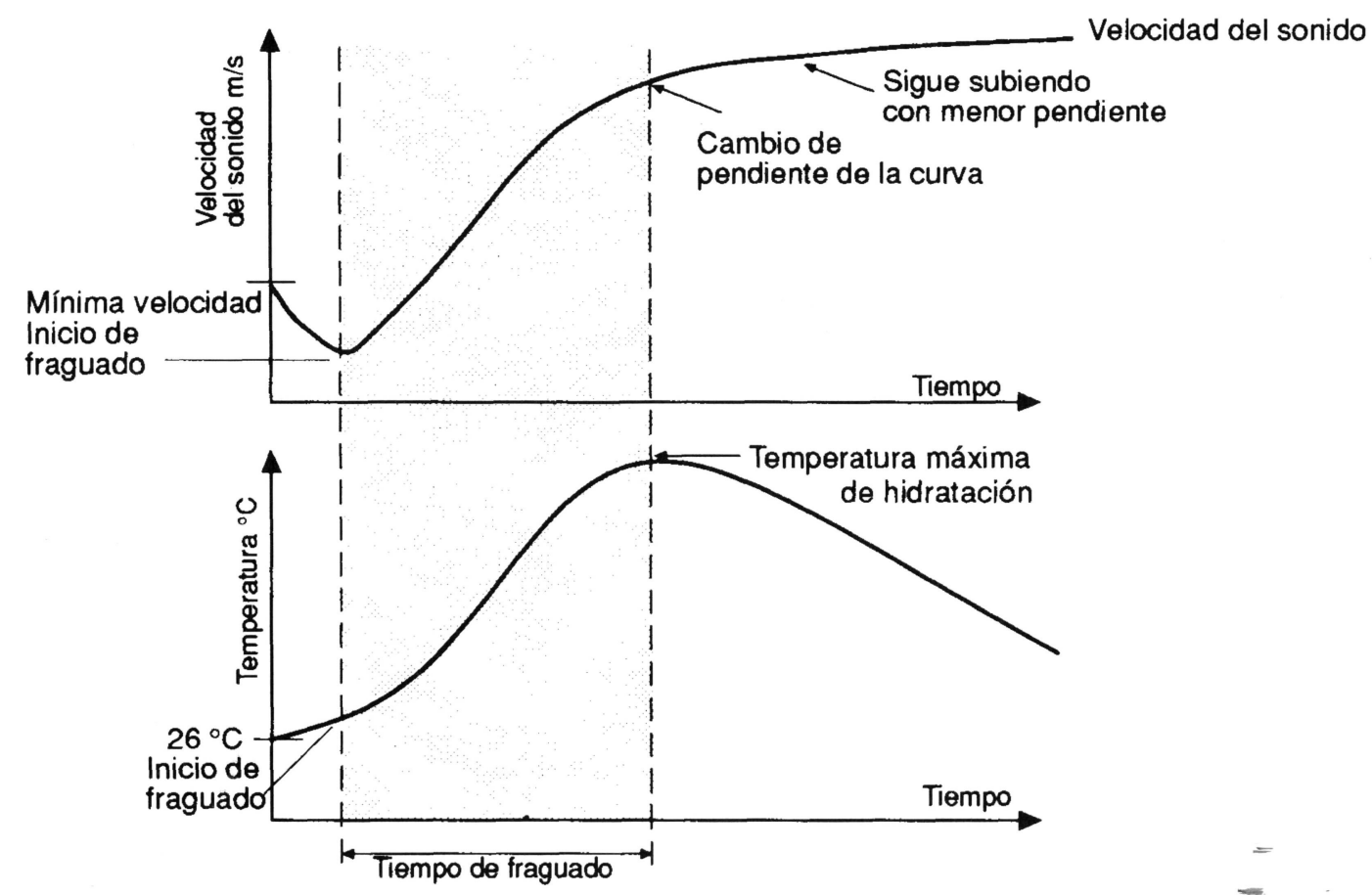

Fig. 10.- Esquema de la relación entre las curvas de velocidad del sonido y la temperatura de hidratāión. 


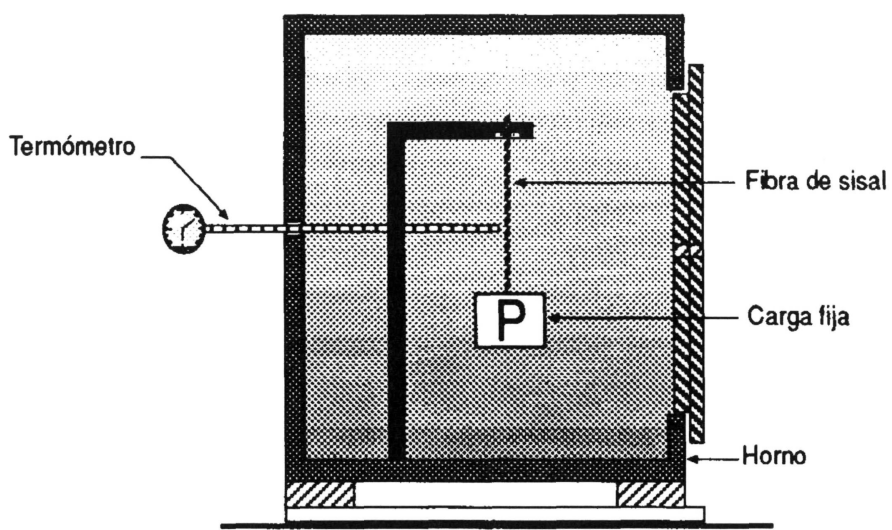

Fig. 11.- Disposición del ensayo de tracción de fibras con aumento de la temperatura.

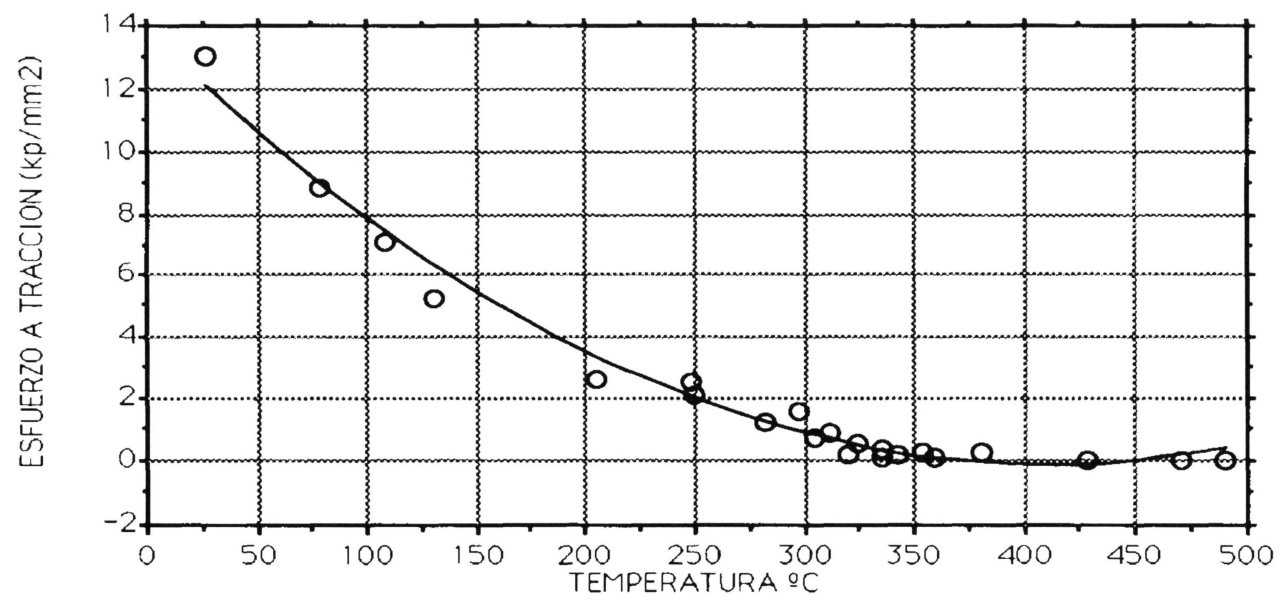

Fig. 12.- Curva de la resistencia a tracción de la fibra de sisal en función de la temperatura.

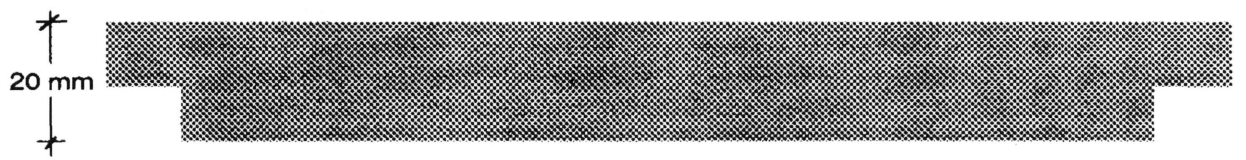

Probeta antes del ensayo

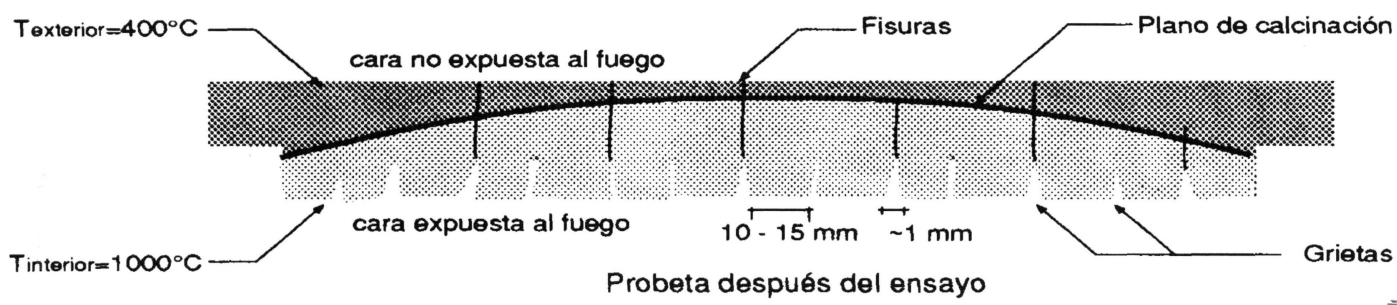

Fig. 13.- Secciones de la probeta, antes y después del ensayo a altas temperaturas. 
- La durabilidad de las fibras vegetales es objeto de diversas investigaciones, en especial al utilizarlas con matrices a base de cemento. Sobre el estudio de la durabilidad de las fibras de sisal en matrices de yeso o de escayola no se conocen trabajos, debido probablemente a la limitación del uso de la escayola para espacios interiores y protegidos del medio ambiente y a su función no estructural en la construcción.

- Debe estudiarse la posibilidad de utilizar componentes a base de yeso o escayola reforzada para muros de viviendas unifamiliares. Uno de los problemas del material, su fragilidad, es posible solucionarlo mediante la adición de fibras como refuerzo. El otro problema, la sensibilidad y disminución de resistencia del yeso ante el agua, es un tema de investigación primordial, si se pretende utilizar este material de forma masiva en viviendas de bajo coste.
- Una línea que se considera importante para investigar, trata del estudio de las aplicaciones concretas del material analizado en este trabajo, con el fin de proponer componentes constructivos para viviendas, que puedan ser de fácil realización como elementos prefabricados, y que permitan un montaje sencillo.

\section{AGRADECIMIENTOS}

El autor desea expresar su gratitud a los miembros del Equipo de Investigación "Alternativas tecnológicas de bajo coste para viviendas de interés social", del Instituto de Ciencias de la Construcción Eduardo Torroja - CSIC y a los miembros del Departamento de Construcción y Tecnología Arquitectónicas de la Escuela Técnica Superior de Arquitečtura de la Universidad Politécnica de Madrid, por su asesoramiento y colaboración en el desarrollo de esta tesis doctoral.

\section{publicación del ICCET/CSIC}

\section{ACUEDUCTOS ROMANOS EN ESPAÑA Carlos Fernández Casado \\ Prof. Dr. Ing. de Caminos, Canales y Puertos}

Esta publicación se compone de una serie de articulos, publicados en la Revista "Informes de la Construcción", en los cuales se hace un análisis de los acueductos romanos que existen en España y el balance de las condiciones de conservación en que se encuentra cada uno de ellos, incluyendo referencias históricas y literarias. Se ha ilustrado con la reproducción de la valiosa documentación gráfica que posee el prestigioso autor.

Un volumen encuadernado en couché, a dos colores, de $21 \times 27$ centímetros, compuesto de 238 páginas, numerosos grabados, dibujos, fotos en blanco y negro y figuras de linea.

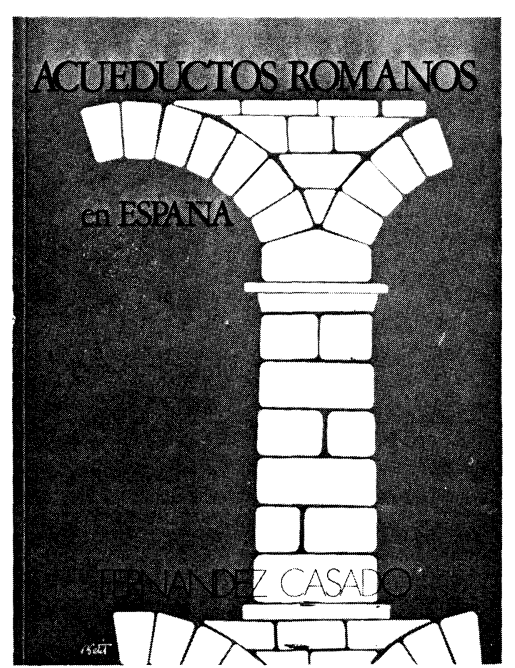

